\title{
Theories on the Formation and Evolution of Black Holes \& Galaxies
}

\author{
James \& Henry Bushong
}

Author contact: M2Materials@Outlook.com

\begin{abstract}
:
Conventional theory suggests that black holes are singularities of enormous mass-density: matter compressed beyond imagination due to extreme mass-based gravitational forces and possessing so much mass-based gravity that light itself cannot escape them. As an alternative to convention, this paper builds on the theories of fire-tornado accretion cylinder vortex forces and colossal magnetic pressure spawned within (previously described by the authors in their paper on $\sim 2 \mathrm{D}$ planar celestial kinematics), and analyzes them in more detail specifically for black holes and the formation / evolution of galaxies. Several interesting charge-distribution and associated electromagnetic field components will be utilized in the modeling. To demonstrate concept, the proposed forces during formation and evolution will be computationally modeled and translated into visual simulations in 4-D space-time using C\# programming in the Unity operating platform.
\end{abstract}

Keywords: black holes, super-massive black holes, galaxy formation, galaxy evolution, nuclear fusion, Z-pinch magnetic compression, electromagnetic fields, celestial kinematics

\section{Background and the Physics of Model Development:}

As discussed in the previous publication "Theories on the Formation and Evolution of $\sim 2 \mathrm{D}$ Planar Celestial Kinematics" , the proposed forces are identical in concept for the formation of stars/solar systems and galaxies except that the force magnitudes and distance over which they act is many times more epic in galaxies than the already colossal forces modeled in the formation of our solar system's sun, and that the proposed fire-tornado vortex and associated forces at the center of the Milky Way and other active galaxies still rages (though at a lower extent today relative to the formation period billions of years ago).

Compared to the sun and solar-system models of the previous paper, it remains even more remarkable that the $\sim 2 \mathrm{D}$ planar celestial kinematics of galaxies like the Milky Way occurred considering the billions of stars and vast distances over which they are in violation of the expected tendency of matter/energy toward spatial homogeneity (i.e. more uniform dispersion of matter / orbital kinematics throughout the vast 3D/4D space-time over which the matter would have expected to occur considering the law of entropy acting over billions of years).

Conventional theory attributes such anomaly to kinetic rotational forces and collapsing due to gravity; wherein the gravity at the central black-hole region is so intense that no known force in nature can withstand it and crushes all matter into a singularity, and that this incredibly dense mass-singularity creates gravity so strong that light cannot escape it within the event-horizon.

As before, the theories herein suggest that the rotating accretion matter that forms galaxies is more aptly modeled as an immense fire-tornado vortex that would have continuously grown in height if not for another powerful force acting to the contrary. With no other place to go, in the absence of other forces a heat-driven vortex with a continuous-energy source would normally cause matter to distribute along the "height axis" due to forces such as increasing pressure and electric repulsion within the tightening-width vortex. The proposed models suggest that there 
was (and continues to be for active galaxies) such concurrent compression force caused by a colossal electric field in the fire-tornado accretion cylinder vortex spawning colossal magnetic field and associated colossal magnetic pressure acting primarily in the $+\hat{Z}$ and $-\hat{Z}$ axis, all rotating about a central-core zone that is mostly void of matter as in the eye of a tornado. This model will be referred to as the NOEMKV (Net Omnipresent ElectroMagnetic Kinetic Vortex) field-theory. This paper will focus on how NOEMKV fields formed and calculate estimates of the strength of magnetic compression in forming the galactic core of the Milky Way, as well as propose interesting related astrophysics theory.

In the proposed solar model, the eye of the solar accretion cylinder vortex becomes what is conventionally referred to as the barycenter of a solar system. However instead of a "center of mass-gravity" that the barycenter of space around which the sun orbits is conventionally referred, our model considers this space to be the remnant of the fire-tornado eye at the final stage of the proposed star-formation process. For the proposed galactic model, the eye of the galactic accretion cylinder vortex becomes a black hole; but instead of an incredibly-dense mass singularity of convention, the proposed black hole model is a region of space essentially void of matter. This matter-void zone is proposed to be a barrier that is naturally built by a rotating vortex, and likely reinforced by either or both tremendous electric and magnetic repulsive forces.

The colossal strength of the electric and magnetic fields (EM fields), associated magnetic pressure, and the source of the proposed tremendous electric and/or magnetic repulsion in the eye of the accretion vortex would be derived from the alignment, distribution, rotational convection, conduction, and displacement of electric charge and the resulting net vectors of EM fields and associated magnetic pressure. This paper will analyze the EM fields in more detail and their effect in particular on the compression of the Milky Way during the formation-process that resulted in the present-day observed $\sim 2$ D planar kinematics $(\sim 100,000$ light years width with height of only $\sim 4,000$ light years).

According to the proposed fire-tornado vortex model, the matter rotating around the inner-core region around the eye will have the greatest velocity and friction-induced heat. Since the velocity and temperature are at a maxima at this region, the wall of matter (plasma) around the eye and a region around the wall should have atoms completely ripped into their constituent electrons, protons, and neutrons. The region of free atomistic constituents would continue to expand as the amount of hottest and highest-velocity matter rotating around the eye of the vortex increases as the vortex intensifies.

An interesting postulate of the models is a mass-based centrifuge-like separation induced by the fire-tornado vortex. As will be detailed below, the proposed mass-based vortex-induced separation would create charge-distribution zones. These charge-distribution zones are proposed to organize and strengthen the electric field, magnetic field, and magnetic pressures, and create ideal conditions for nuclear fusion which would generate additional energy to drive the vortexengine. Beyond the fundamental understanding of this research project, it is proposed that mimicking these conditions to the highest practical extent could increase humanity's chances of harnessing the power of nuclear fusion as an energy source. There may also be practical uses for utilizing the vortex-created magnetic pressure methods described herein as a source of power for motors and engines- including a potential alternative to combustion processes for rocket engines. 
The proposed vortex-induced centrifuge-like mass-based separation and associated charge distribution can be modeled as charge-rich cylindrical shells rotating around the eye. Two interesting charge-distribution models are considered for this study:

\section{Charge-Distribution Model 1}

In this model, heavier protons and neutrons are organized into an inner-most cylindrical shell around the eye and the much smaller-mass electrons are organized into another cylindrical shell around the proton/neutron cylindrical shell. This will create a strongly positive-charge-rich shell surrounded by a strongly negative-charge-rich shell. The electric field is predicted to have radial components (starting from the positive-charge-rich shell directed radially outward toward the negative-charge-rich shell in a conduction current) and azimuthal rotational components in the $\theta$ plane around the height $(\hat{Z})$ axis due to a convection-displacement current created by the continuously and rapidly rotating charged cylindrical shells (with rotational velocity of the outer shell matter being less than that of the inner shell matter in the vortex). The charge distribution and predicted electric field components of this model are depicted as:

\section{Figure 1: Charge-Distribution Model 1}

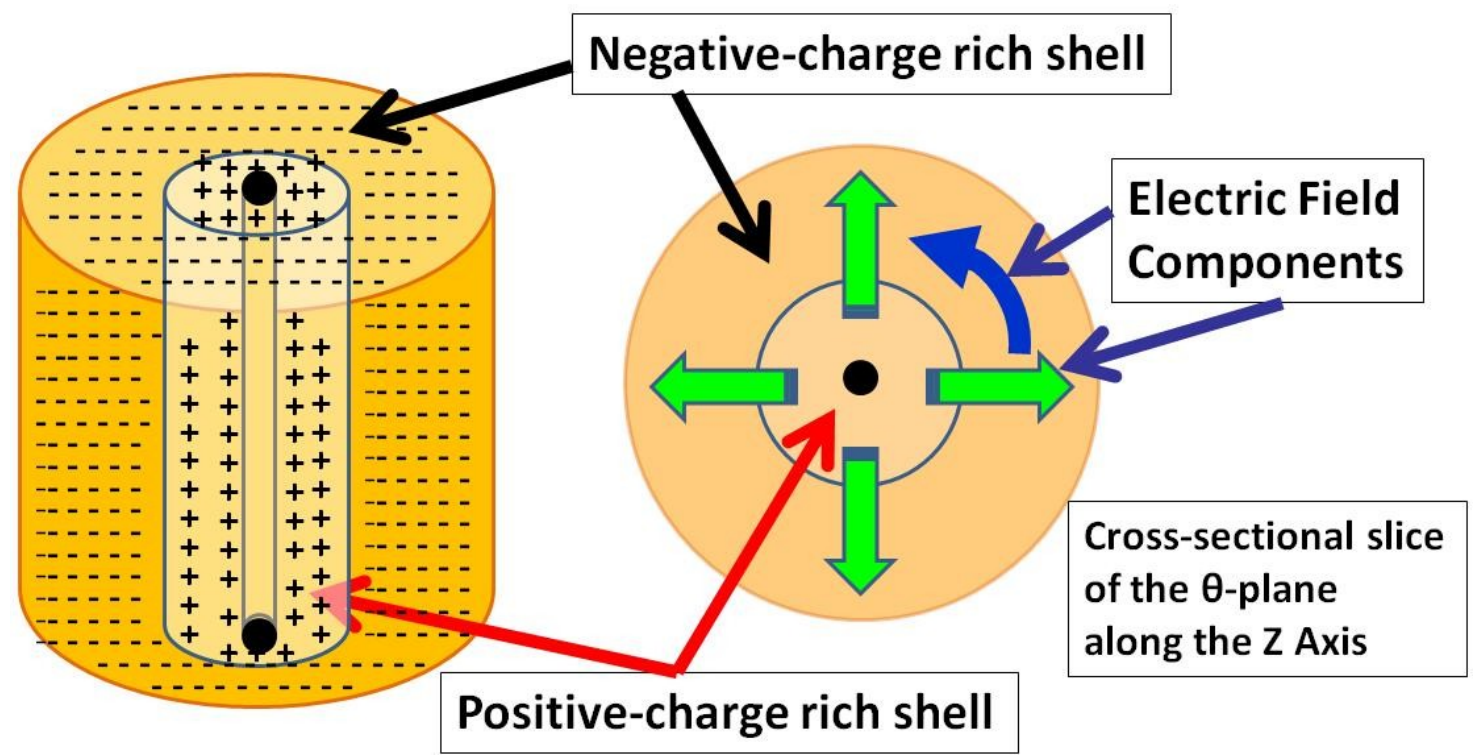

The net vector of the electric field with the two components shown would likely be a continuous spiraling field originating at the positive-charge-rich cylindrical shell and spiraling outward from there to the negative-charge-rich cylinder, for each differential cross-section in the $\theta$-plane along the $\mathrm{Z}$ axis. The spawned magnetic field would mimic the continuous spiraling electric field though orthogonally at every point at any instant in accordance with electromagnetic theory. 


\section{Charge-Distribution Model 2}

This model is the same basic concept as Model 1, except that it adds the potential effects at the boundary conditions:

- At the "top and bottom faces" of the accretion-cylindrical vortex, heat transfer between these boundaries and the extreme coldness of space can slow the rotational speed and decrease the overall kinetic energy and pressure of the rotating gas/dust/plasma.

- At the outer sidewalls of the accretion vortex, the velocity is slower (typically decreasing as $1 / \mathrm{r}$ from the center of the vortex), and again there is considerable heat-transfer between the outer gas/dust/plasma and the coldness of space to further decrease the overall kinetic energy and pressure of the rotating gas/dust/plasma

- At these boundary face regions, electrons are modeled as remaining with their constituent atomic nuclei (referred to herein as "wholistic atoms")

In this model, electric field components would exist from radial conduction (from the positivecharge-rich shell radially outward toward the negative-charge-rich shell and then to a conventional induced-dipole arrangement of charge in the wholistic atoms of a third cylindrical shell from the sidewall-boundary to the negative-charge-rich shell), transverse conduction (moving along the $+\hat{Z}$ and $-\hat{Z}$ axis all along the inner boundary wall between the positivecharge-rich shell/eye of the fire-tornado vortex and ending at an induced-dipole in the wholistic atoms at the top and bottom face boundary regions), and an azimuthal displacement-convection current due to the continuous and rapid rotation of charges within each of the three cylindrical shells (each of which is modeled as rotating at a slower velocity as distance from the center of the vortex increases). The charge distribution and predicted electric field components of this model are depicted as:

\section{Figure 2: Charge-Distribution Model 2}

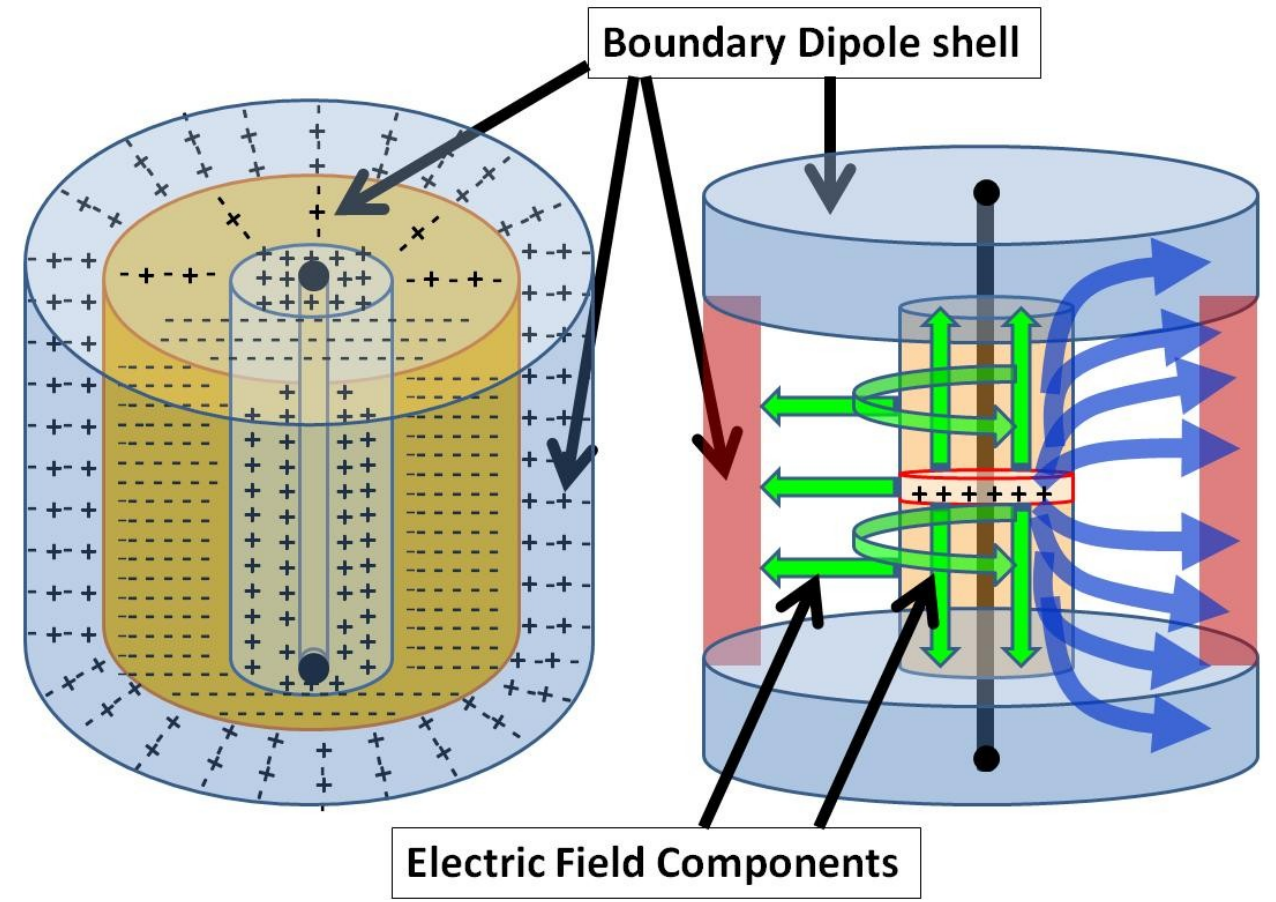


In Figure 2, the two inner cylindrical shells are identical in concept to Model 1, with the addition of the shell of induced-dipole of wholistic atoms all around the outside of the negative-rich shell due to the prescribed boundary conditions. The depiction on the right side shows the top and bottom boundaries (blue cylinders with the black hole / tornado eye protruding through the center), a longitudinal-slice of the sidewall boundaries in dark red, the inner positive-charge rich cylindrical shell (in light orange color) from which the electric field lines originate, the radial, transverse, and azimuth-rotational components of the electric field with the green arrows, and approximation of the net vector electric field of these two components (in the longitudinal planeslice) with the blue arrows.

A cross-sectional slice (white with red border lines) in the $\theta$-plane of the inner shell is shown at the midpoint in the $\mathrm{Z}$ axis, from which it is proposed the electric field diverges from this $\mathrm{Z}$ central plane toward the "top face" of the accretion cylinder above this point, and diverges toward the "bottom face" below this point. The third component of the electric field, that of an azimuthally-rotating field all around the $\mathrm{Z}$ axis in the $\theta$-plane, would be the same in concept to Model 2 except with the addition of the third-cylindrical shell of induced-dipole wholistic atoms rotating at a slower velocity than the inner cylindrical shells and adding additional strength to the electromagnetic field with conduction and displacement-convection current. The proposed net vector of the electric field would be one that continuously spirals outward just as in Model 1, though with net direction also having components in the $+Z$ axis (above the $Z$-midpoint) and $-Z$ axis (below the Z-midpoint).

Tracing one electric field line from the Z-midpoint and higher would be a spiral that moves both radially outward and transversely upward at the same time, and tracing one electric field line from below the Z-midpoint would spiral radially outward and transversely downward at the same time. This would appear as two helixes, one on top of the other; both starting with smallest diameter around the positive-charge-rich shell at the Z-central plane, growing in diameter as the field line moves transversely along the Z-axis, and ending with the largest diameter around the outer induced-dipole shell at the top and bottom boundary faces of the accretion cylinder. The spawned magnetic field would mimic the continuous spiraling electric field though orthogonally at every point at any instant in accordance with electromagnetic theory.

Both charge-distribution models would have an un-naturally large positive charge around the wall-forming the eye of the vortex due to the centrifuge-charge separation effect, and any part of the wall around the eye will have strong electric repulsion to all other parts of the wall. This strong repulsion is proposed to reinforce the strength of the inner wall formed naturally around the eye of the vortex-engine, and help prevent collapse. Although this eye region is proposed to be essentially void of mass (for both the solar and galactic models), the existence of this inner wall of the accretion-cylinder vortex around empty space likely plays a vital role in the orientation and direction of the NOEMKV field as a surface from which EM fields can converge, diverge, or rotate in 4-D space-time. Strong electric and/or magnetic repulsion at the inner wall could be responsible for intense expulsion of any matter that crosses the eye-region; such crossing could for example occur at the top and bottom surface boundary layers where the vortex is weakened due to heat-transfer with cold space.

Computer animations of the 4D dynamic process including azimuthal rotation and compression in the Z-axis of the galactic accretion vortex during formation will be completed as part of this study. The magnetic field lines originating from the net electric field will be of particular interest 
in the calculations and animations, since it is the magnetic field strength that will dictate the magnitude and direction of compression forces proposed to play a large role in the formation of $\sim 2$ p planar galaxies.

\section{Equations and Computational Algorithm Models}

All of the mathematical and computational modeling in the preceding paper will now be applied to the Milky Way, beginning with the development of the kinematic construct. The starting point of the kinematic construct for the fire-tornado vortex of the Milky Way is a diagram from NASA. ${ }^{2}$ This diagram shows an elliptical region of intense light at the center of the galaxy (labeled as the "Galactic Bar") from which the arms of spiral arms extend, a common feature among active spiral galaxies. The diagram further shows an even more intense circular core region. For purposes of the initial kinematic construct, the very intense circular core region is selected as the primary fire-tornado vortex; this is believed to coincide with the region referred to as the "bulge" of the Milky Way, a flattened spherical region of intensely hot matter at the center of our galaxy. Utilizing the Light-Year (LY) scale of this diagram, the initial galactic kinematic construct is:

\section{Figure 3: Kinematic Construct of the Milky Way Fire-Tornado Vortex Model}

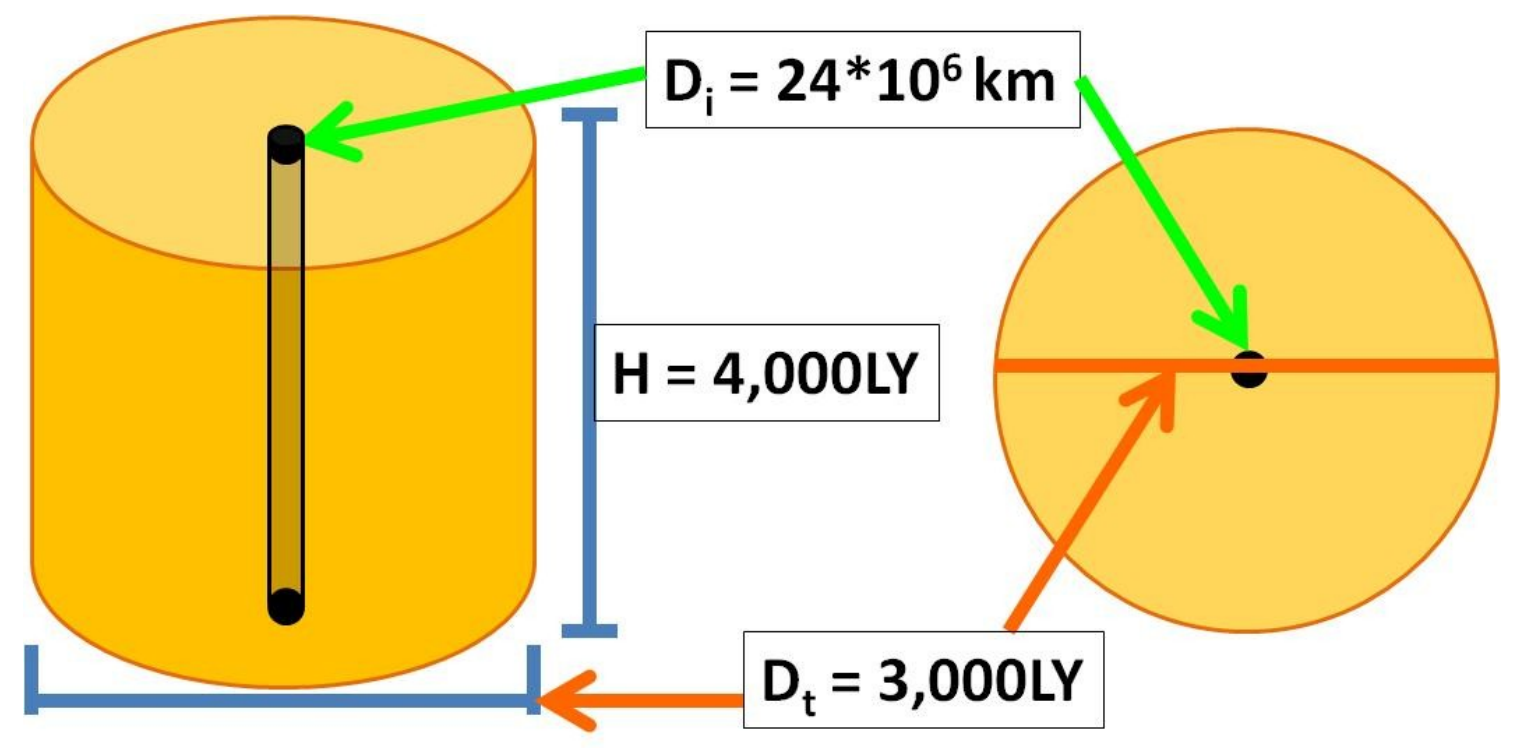

In Figure 3, the height of the cylinder is modeled as the height of the "thick disk" of the Milky Way $(\sim 3,500 \text { light years })^{3}$ plus 250 light years on either side of the galactic plane to account for the "bulge" at the Milky Way core. In the figure, the size of the black hole is exaggerated to show its existence, estimated to be about $24 * 10^{6} \mathrm{~km}$ in diameter ${ }^{4}$ which although is a relatively large number at 17 times the diameter of our Sun is tiny in comparison to the 3,000 light-year diameter $\left(2.8383 * 10^{16} \mathrm{~km}\right)$ very-intense primary circular galactic accretion cylinder vortex utilized in this model.

The computational algorithms from the previous publication on $\sim 2 \mathrm{D}$ planar celestial kinematics apply; their derivations will not be repeated except where modifications have been made. The 
algorithms will be utilized to estimate the respective parameters of NOEMKV for galactic formation and evolution of the Milky Way, as follows:

Volume of Galactic Accretion Cylinder kinematic construct:

\section{$\mathrm{V}=\pi^{*}\left(\mathrm{R}_{\mathrm{t}}^{2}-\mathrm{R}_{\mathrm{i}}^{2}\right) * \mathrm{H}=\pi^{*}\left(\mathrm{R}_{\mathrm{t}}^{2}\right)^{*} \mathrm{H} \quad($ Equation 1)}

Where:

$\mathbf{R}_{\mathbf{t}}=$ total radius of the cylinder

$\mathbf{R}_{\mathbf{i}}=$ inner radius of the cylinder (eye of the fire-tornado / the black hole)

$\mathbf{H}=$ cylinder height

And the equation reduces to the simplified form shown because in this case $\mathrm{R}_{\mathrm{i}}<<\mathrm{R}_{\mathrm{t}}$

Next, since the density of matter in the galactic accretion cylinder is problematic to determine, the assumption is made that the state of matter in this galactic region is equivalent to that at the end of Stage 3 of the star formation process detailed in the previous publication (the peak of the NOEMKV field forces for star formation). This assumption is made by modeling the active galactic core matter as very similar to that stage since fuel is still available for active galaxies to drive the vortex-heat engine and cooling/coalescence into star-like density has not yet occurred at their core. The state of matter at the end of Stage 3 of the star-formation process resulted in densities $\left(\rho_{\max }\right)$ of $6.7 * 10^{10}-8.3^{*} 10^{10} \mathrm{~kg} / \mathrm{km}^{3}$ at $\mathrm{H}_{\text {min }}$ for Models 1 and 2 in the previous publication. This paper will assume the average of these, so $\rho_{\max }$ is assumed to be $7.0^{*} 10^{10}$ $\mathrm{kg} / \mathrm{km}^{3}$ for the galactic accretion cylinder density at $\mathrm{H}_{\min }(4,000 \mathrm{LY})$; this is assumed to be the density and height of the galactic accretion cylinder model at the end of the primary formation process of the Milky Way's core billions of years ago and continuing to this day.

With Volume and Density in-hand, an estimate of the mass of the galactic accretion cylinder can easily be calculated. These values are summarized as:

Table 1: Numeric Summary of Galactic Kinematic Construct (Present-day and at the end of Primary-Formation process)

\begin{tabular}{|c|c|c|c|c|}
\hline $\begin{array}{c}\text { Height } \\
(\mathrm{km})\end{array}$ & $\begin{array}{c}\text { Diameter } \\
(\mathrm{km})\end{array}$ & $\begin{array}{c}\text { Volume of } \\
\text { Galactic-Core } \\
\text { Cylinder }\left(\mathrm{km}^{3}\right)\end{array}$ & $\begin{array}{c}\text { Density } \\
\left(\mathrm{kg} / \mathrm{km}^{3}\right)\end{array}$ & $\begin{array}{c}\text { Mass of } \\
\text { Galactic Core } \\
(\mathrm{kg})\end{array}$ \\
\hline $3.784 \mathrm{E} 16$ & $2.838 \mathrm{E} 16$ & $2.394 \mathrm{E} 49$ & $7.000 \mathrm{E} 10$ & $1.676 \mathrm{E} 60$ \\
\hline
\end{tabular}

Utilizing these constraints and assuming that the primary galactic cylinder vortex diameter stayed constant from the end of the primary-formation process to the present day, the equation for volume of central accretion cylinder vortex as a function of height is derived to model the pre-maximum compression period (e.g. directly analogous to the NOEMKV field of the starformation model from the end of Stage 2 up until the max-compression NOEMKV field at the end of Stage 3). This will be demonstrated in the Results section.

As in the star-formation model, the next step is calculating the differential magnetic pressure to compress the cylinder of accretion matter at each step of the Stage-3 process, utilizing the equation: 


\section{$\Delta \boldsymbol{P}_{\boldsymbol{m}}=-\left(\frac{\Delta \boldsymbol{V}(\boldsymbol{h})}{\boldsymbol{V}_{o}}\right) * \boldsymbol{K} \quad$ (Equation 2)}

Where:

$\Delta \mathbf{P}_{\mathbf{m}}=$ the change in magnetic pressure generated by the magnetic field $\left(\mathrm{N} / \mathrm{m}^{2}\right)$ at each step of the compression process

$\Delta \mathbf{V}(\mathbf{h})=$ change in volume of the galactic accretion cylinder from one height to the next $\mathbf{V}_{\mathbf{0}}=$ initial volume at each step of the height compression

$\mathbf{K}=$ the Bulk Modulus of the galactic accretion matter $\left(\mathrm{N} / \mathrm{m}^{2}\right)$

The Bulk Modulus $(\mathrm{K})$ of the galactic accretion matter is expected to change over time as the density of the accretion matter within the accretion cylinder continues to increase with the proposed magnetic pressure compression. To obtain accurate values of $\mathrm{P}_{\mathrm{m}}$ would require accurate values of $\mathrm{K}(\rho)$ which is problematic just as in the star-formation model; nevertheless the main point of these analysis are to highlight the concepts and propose the basic force equations with calculations where possible. As such, the key factors of $\mathrm{K}(\rho)$ for the proposed models are assumed to be that of the state-of-matter in the star formation models:

- $\mathrm{K}(\rho)$ maximum $\left(\mathrm{K}_{\max }\right)$ of the galactic accretion cylinder matter will be assumed to be that of experimentally and theoretically-proposed values for the bulk modulus of Lithium one-component plasma ${ }^{5,6}$

- Each subsequent $\mathrm{K}(\rho)$ for all other compressions of galactic accretion cylinder height will be calculated by multiplying the ratios of density between each incremental step of expansion (going further back in time) from the maxima compressed cylinder $\left(\mathrm{H}_{\min }\right)$. Mathematical details are described in the Results section.

The final compression-equation of the galactic model then becomes calculating the magnetic field strength associated with the magnetic pressure. The component of magnetic force acting in the $+\hat{Z}$ and $-\hat{Z}$ directions would be ${ }^{7}$ :

$P_{m}=\frac{B^{2}}{2 \mu_{o}} \quad$ (Equation 3)

Where:

$\mathbf{P}_{\mathbf{m}}=$ magnetic pressure $\left(\right.$ Pascals,$=$ Newtons $\left./ \mathrm{m}^{2}\right)$

$\mathbf{B}=$ Magnetic Field Strength in Teslas

$\boldsymbol{\mu}_{\mathbf{0}}=$ magnetic permeability of free space $=4 * \pi * 10^{-7}$ Henrys $/$ meter

\section{Results \& Discussion}

The results of the computational algorithms and implications on magnetic fields and associated magnetic pressure will now be discussed. Modeling of charge distribution and the net-vector of magnetic fields derived from the electric-field components will be performed supplementary with 4-D space-time simulations in C\# code in the Unity operating platform. 
Values for $V(h)$ and $\rho(h)$ for the galactic accretion cylinder starting at $\mathrm{H}_{\text {min }}$ were calculated using float values for $\mathrm{H}$ in iterative loop scripts of Equation 1 written in $\mathrm{C} \#$ in the Unity. Starting at $\mathrm{H}_{\text {min }}$, step gain increments were:

$9.461 * 10^{14} \mathrm{~km}(100 \mathrm{LY})$ for 20 steps up to 1.5 times $\mathrm{H}_{\text {min }}$ $4.731 * 10^{15} \mathrm{~km}(500 \mathrm{LY})$ for 18 steps up to 3.75 times $\mathrm{H}_{\text {min }}$ $4.731 * 10^{16} \mathrm{~km}(5,000 \mathrm{LY})$ for 21 steps up to $\sim 30$ times $\mathrm{H}_{\min }$

The increase in step value was chosen to keep the data for analysis in Excel to a reasonable level, while ensuring sufficient data at all regions of plotted data to show clear trends without ambiguity (noting that for visual computational simulations all functions are essentially infinitely continuous as there are relatively no processing power limitations with frame-rate for the equations utilized in this modeling). The end point of maximum height was selected to correspond with the current estimated galactic width $(\sim 120,000$ Light Years). The results are plotted in Figure 4A:

\section{Figure 4A: Galactic Accretion Matter Density as a Function of Cylinder Height}

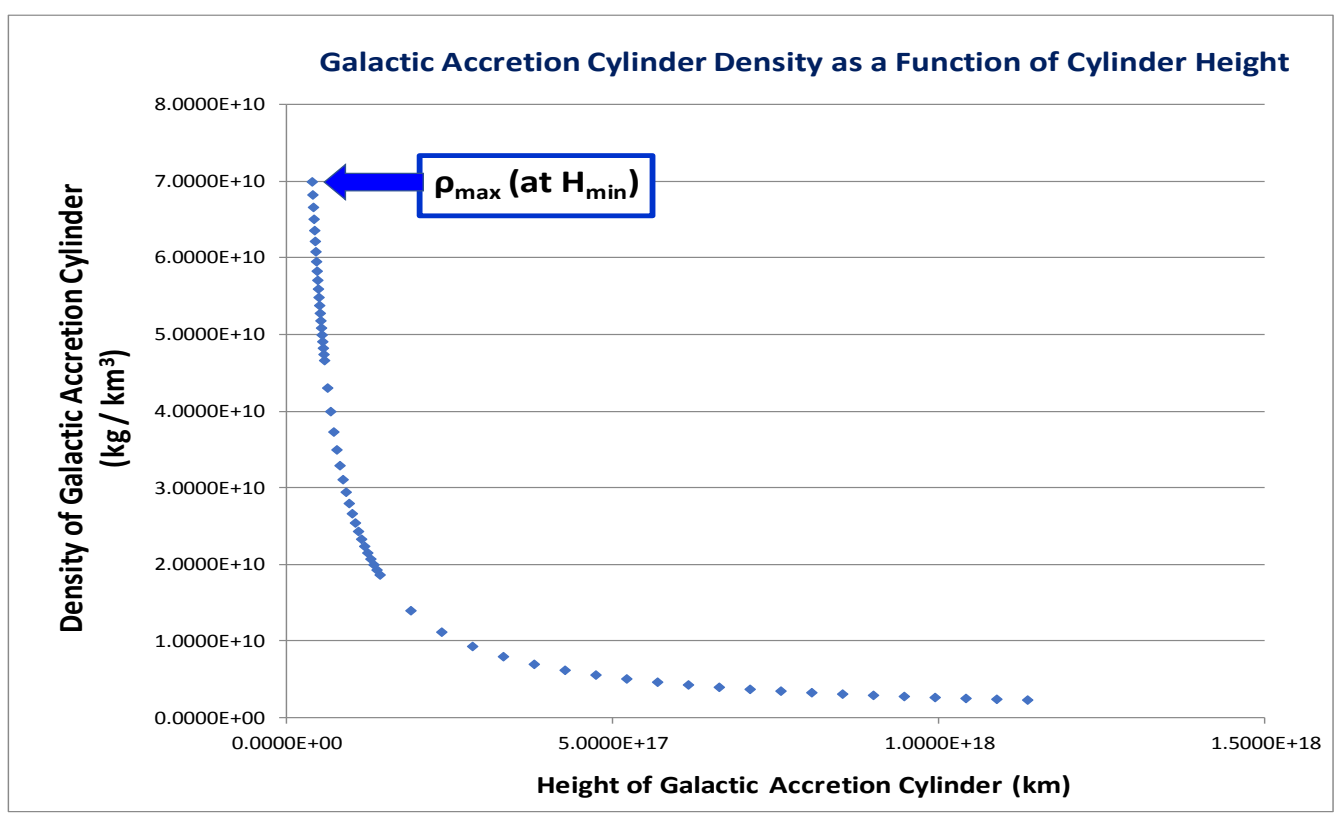

As in the star-formation models, the galactic models are focused on the density increase associated with the organization of gas/dust/plasma from the kinetic forces of a fire-tornado vortex and subsequent magnetic pressure compression, so it is desirable to find the point in $\mathrm{H}$ at which the rate of density change begins to substantially increase. This is determined by plotting the $2^{\text {nd }}$ derivative of the $\rho(\mathrm{h})$ equations vs. $H$, to elucidate where the rate of change of the $\rho$ vs $H$ slope begins to noticeably change. The precise equations for $\rho(\mathrm{h})$ in $\mathrm{kg} / \mathrm{km}^{3}$ for the Milky Way galactic model and the second derivative is: 


$$
\begin{aligned}
& \rho=\frac{2.648 * 10^{27}}{H} \\
& \frac{d^{2} \rho}{d h^{2}}=\frac{5.296 * 10^{27}}{H^{3}}
\end{aligned}
$$

With results plotted numerically in Figure 4B:

\section{Figure 4B: Second-Derivative of $\rho$ vs $H$ for the Galactic Accretion Cylinder}

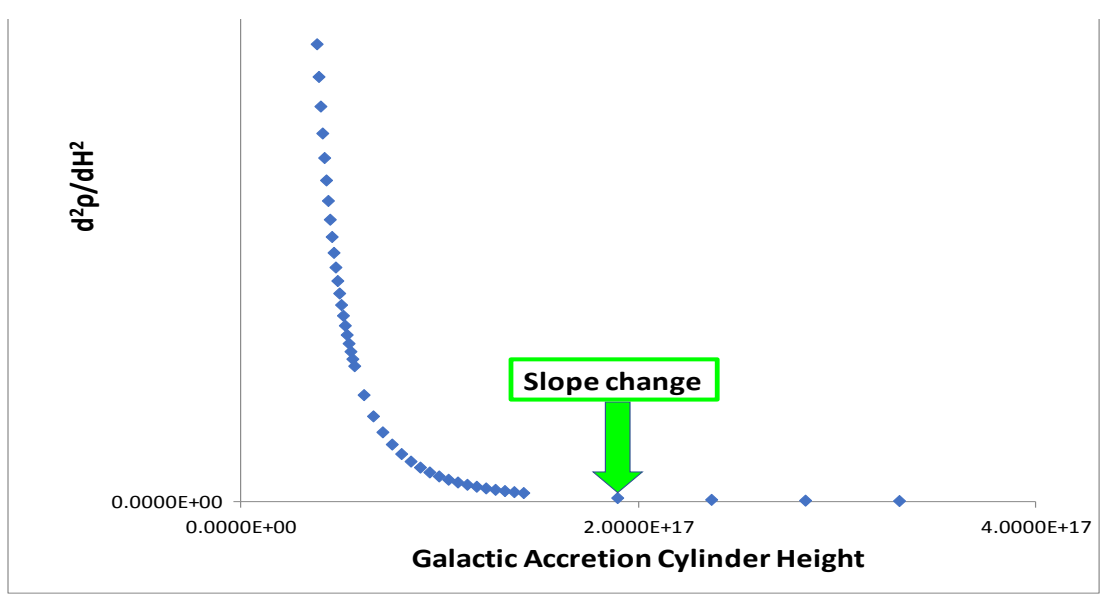

The point at which the slope of the $2^{\text {nd }}$ derivative substantially increases will be assumed to be the point of galactic accretion cylinder height with sufficient organization to meet the following constraint-criteria:

- The central vortex diameter (eye of the fire tornado) achieves equivalence with the current diameter of the black hole (Sagittarius A*) at the center of the Milky Way

- The diameter of the primary galactic vortex at the core of the Milky Way during the formation process was equivalent to the current diameter of the most-intense heat and velocity region at the core of the present-day Milky Way (assumed to be the "bulge" of the Milky Way of the kinematic-construct parameters shown in Figure 3 and Table 1)

For the Milky Way, this point is selected from Figure $4 B$ as the data point $\mathbf{H}_{\mathbf{c}}=\mathbf{1 . 8 9 2 2} * \mathbf{1 0}{ }^{\mathbf{1 7}} \mathbf{k m}$ where $\mathbf{H}_{\mathbf{c}}=$ critical vortex height, and marks the end of Stage 2 of the primary formation process (where the kinetic and heat-driven forces of the fire-tornado provide organization of matter around an eye of a vortex) and beginning of Stage 3 (where the strength of the magnetic field and magnetic pressure compress the organized matter into $\sim 2 \mathrm{D}$ planar kinematics) of the Milky Way's formation according to the NOEMKV model conventions previously described in the star-formation models and publication.

A computational script in $\mathrm{C} \#$ was then written to calculate the change in volume $(\Delta \mathrm{V})$ between each incremental step of compression (incremental decrease in cylinder height $H$ from $h_{o}$ to $h_{1}$ )) relative to the initial volume $\left(\mathrm{V}_{\mathrm{o}}\right)$ at the start of that compression step $\left(\mathrm{at} \mathrm{h}_{\mathrm{o}}\right)$. Because Equation (2) is for compression, care is taken in calculating the difference such that the volume at the end of the step $\left(\mathrm{V}_{1}\right)$ is the lower (more compressed) volume compared to that at the start of the step $\left(\mathrm{V}_{\mathrm{o}}\right)$. Mathematically, for the proposed model this becomes: 
$\frac{\Delta V}{V_{o}}=\frac{\left(\pi\left(R_{t}^{2}\right) * h_{1}\right)-\left(\pi\left(R_{t}^{2}\right) * h_{o}\right)}{\pi\left(R_{t}^{2}\right) * h_{o}}$

And since $R_{t}$ is assumed to stay constant during Stage 3, this reduces to:

$$
\frac{\Delta V}{V_{o}}=\frac{\left(h_{1}-h_{o}\right)}{h_{o}} \quad(\text { Equation } 4)
$$

Next is a computational C\# script for calculating bulk modulus $(\mathrm{K})$ as a function of galactic accretion cylinder density $K(\rho)$. The same logic of the star-formation calculations is utilized for the $\mathrm{C \#}$ script for $\mathrm{K}(\rho)$ over all time periods (cylinder heights) from the maximum-compression / maximum-density values of the galactic accretion cylinder to the height at the beginning of Stage 2 assumed to mark the start of the fire tornado intensification phase $\left(\mathrm{H}_{\mathrm{f} 0}\right)$ :

\section{$K(\rho)=\frac{\rho_{o}}{\rho_{1}} * K_{1} \quad($ Equation 5)}

Where:

$\boldsymbol{\rho}_{1}=$ the solar accretion cylinder density after the compression step

$\boldsymbol{\rho}_{\mathbf{0}}=$ the solar accretion cylinder density at the start of the compression step

$\mathbf{K}_{\mathbf{1}}=$ the solar accretion cylinder bulk modulus after the compression step

Executing the computational scripts for $\mathrm{K}(\rho)$ between $\mathrm{H}_{\min }$ and $\mathrm{H}_{\mathrm{f} 0}$ results in:

Figure 5: Bulk Modulus of Galactic Accretion Matter as a Function of Cylinder Height

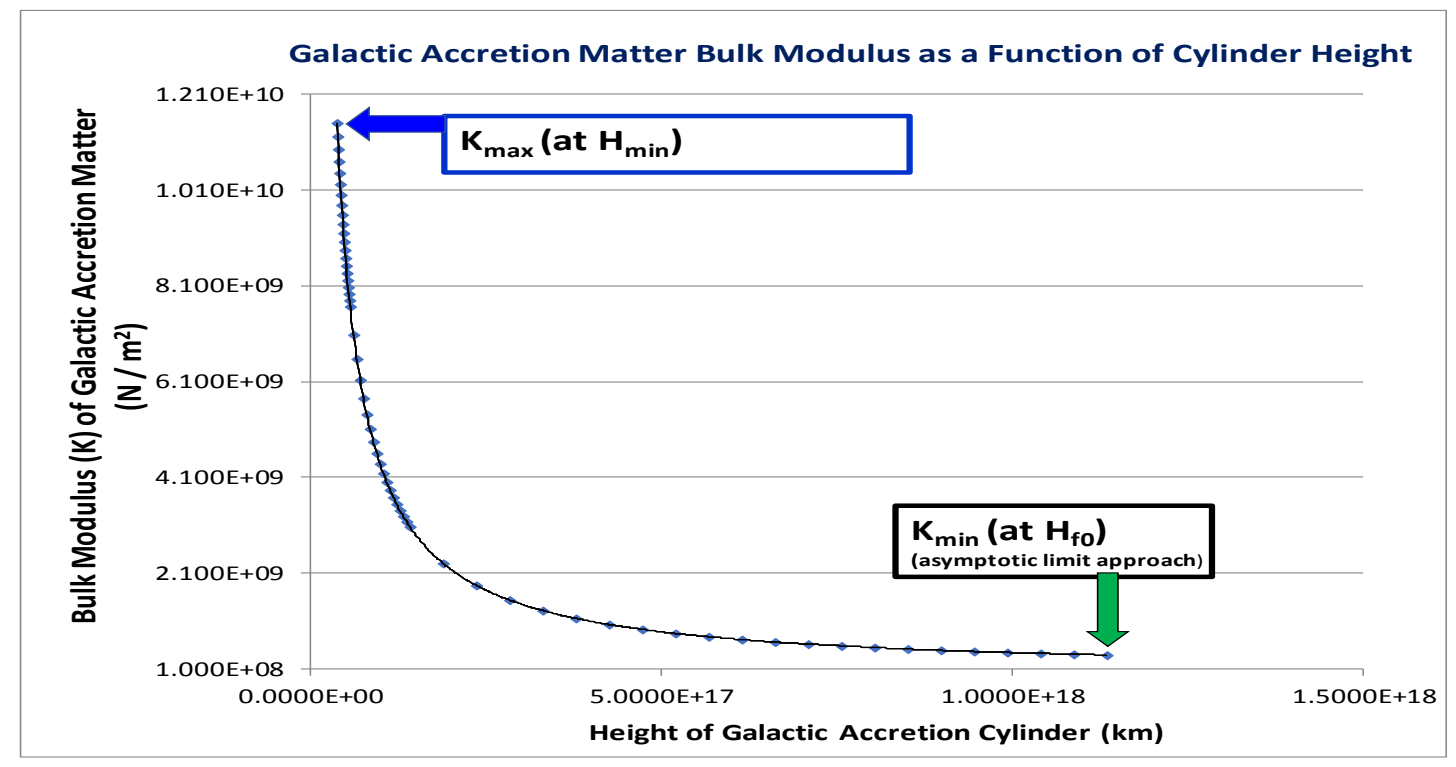

With all the needed parameters of Equation 2 now calculated, the force (differential magnetic pressure in these models) needed to compress the galactic accretion cylinder at each step change of $\mathrm{H}$ was calculated in another $\mathrm{CH}$ script. The cumulative total pressure from the start of the compression process (at $\mathrm{H}_{\text {fo }}$, selected to be $1.135 * 10^{18} \mathrm{~km}$ from the asymptotic limit approach to $K_{\min }$ seen in Figure 5) to the end of Stage $3\left(K_{\max }, \rho_{\max }, P_{\max }\right.$, and $\left.H_{\min }\right)$ of the 
formation of the Milky Way's core was determined by adding the differential pressure at each stage of compression, and is plotted as:

Figure 6: Cumulative Magnetic Pressure as the core of the Milky Way compressed during Stage 3 of Galactic Formation

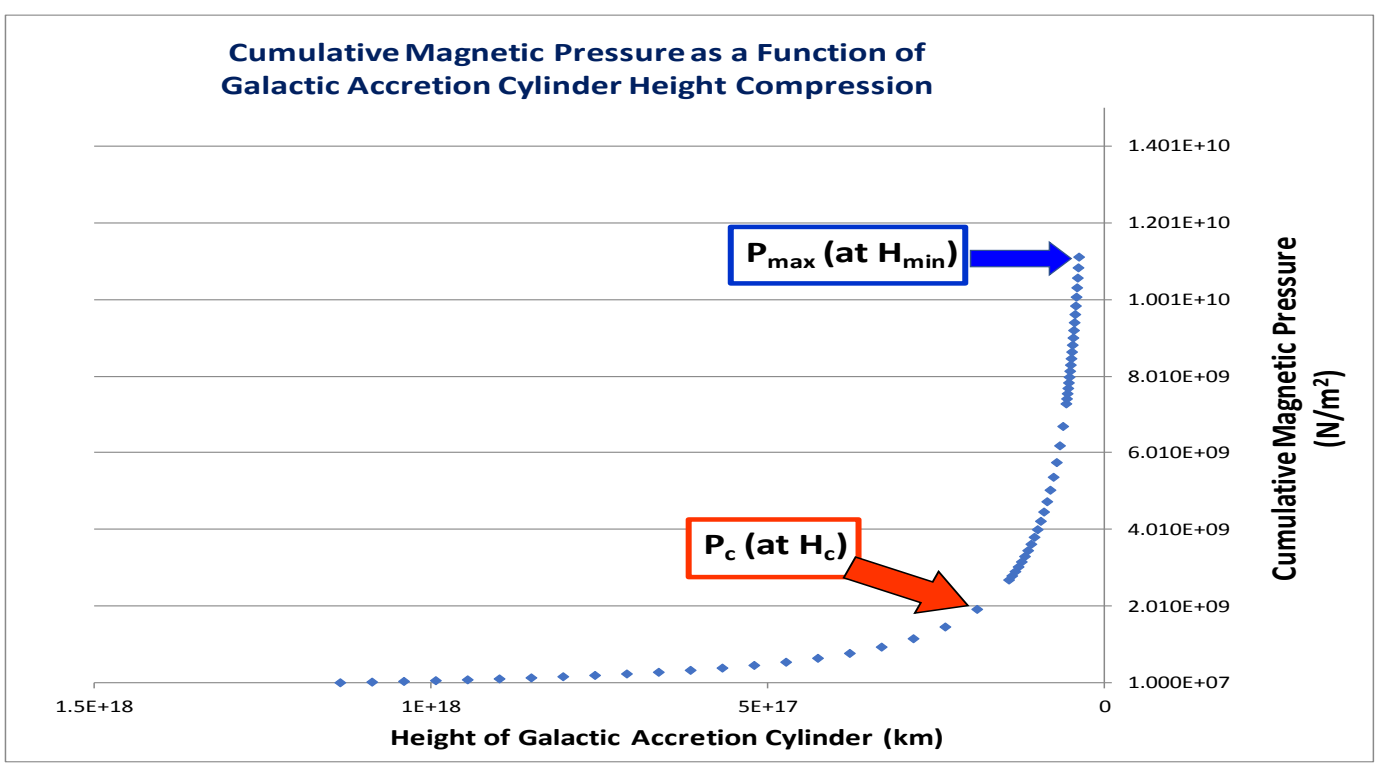

As in the solar-formation model, the cumulative build-up in magnetic pressure is best presented as increasing time (decreasing $\mathrm{H}$ ) as shown in Figure 6. Previous plots were presented as reverse time (increasing $\mathrm{H}$ ) to emphasize that it was the most recent projected data point (at the end of Stage 3 ) at which the calculations started, for reasons as indicated in the development of the final Stage 3 kinematic construct derived from the present-day kinematic construct of the most-intense region of the galactic core. Presenting the data in reverse time was useful for emphasizing that point and for projecting where the start of the fire-tornado organization of galactic accretion matter started (i.e. by choosing a point that began to approach asymptotic lower limit $\mathrm{H}_{\mathrm{fo}}$ ) and where it could be modeled as reaching maximum vortex organization at the critical vortex height $\left(\mathrm{H}_{\mathrm{c}}\right)$ after which time the magnetic field strength (and critical magnetic pressure, $\mathbf{P}_{\mathbf{c}}$ at $\mathbf{H}_{\mathbf{c}}$ shown in Figure 6) rapidly builds and compresses the galactic accretion cylinder. From here on, the more familiar increasing time (decreasing $\mathrm{H}$ ) will be utilized; this will also be helpful and more effective in the visual 4D simulation demos and associated script code.

The colossal magnetic pressures acting in the $+\hat{Z}$ and $-\hat{Z}$ calculated and displayed in Figure 6 are utilized to estimate the magnetic field by rearranging Equation 3 to:

\section{$B_{T}=\sqrt{2 * \mu_{o} * P_{m c}} \quad$ (Equation 6)}

Where:

$\mathbf{B}_{\mathbf{T}}=$ the cumulative magnetic field strength (in Teslas) summed over each stage of compression

$\mathbf{P}_{\mathbf{m c}}=$ the cumulative magnetic pressure (in $\mathrm{N} / \mathrm{m}^{2}$ ) summed over each stage of compression

$\boldsymbol{\mu}_{\mathbf{0}}=$ magnetic permeability of free space $=4 * \pi * 10^{-7}$ Henrys $/$ meter 
The results of $\mathrm{C \#}$ scripts written for calculating cumulative magnetic field strength and then relating that strength to the sun's current magnetic field are shown in Figures 7A and B:

Figure 7A: Cumulative Magnetic Field Strength $\left(B_{T}\right)$ as the core of the Milky Way compressed during Stage 3 of Galactic Formation

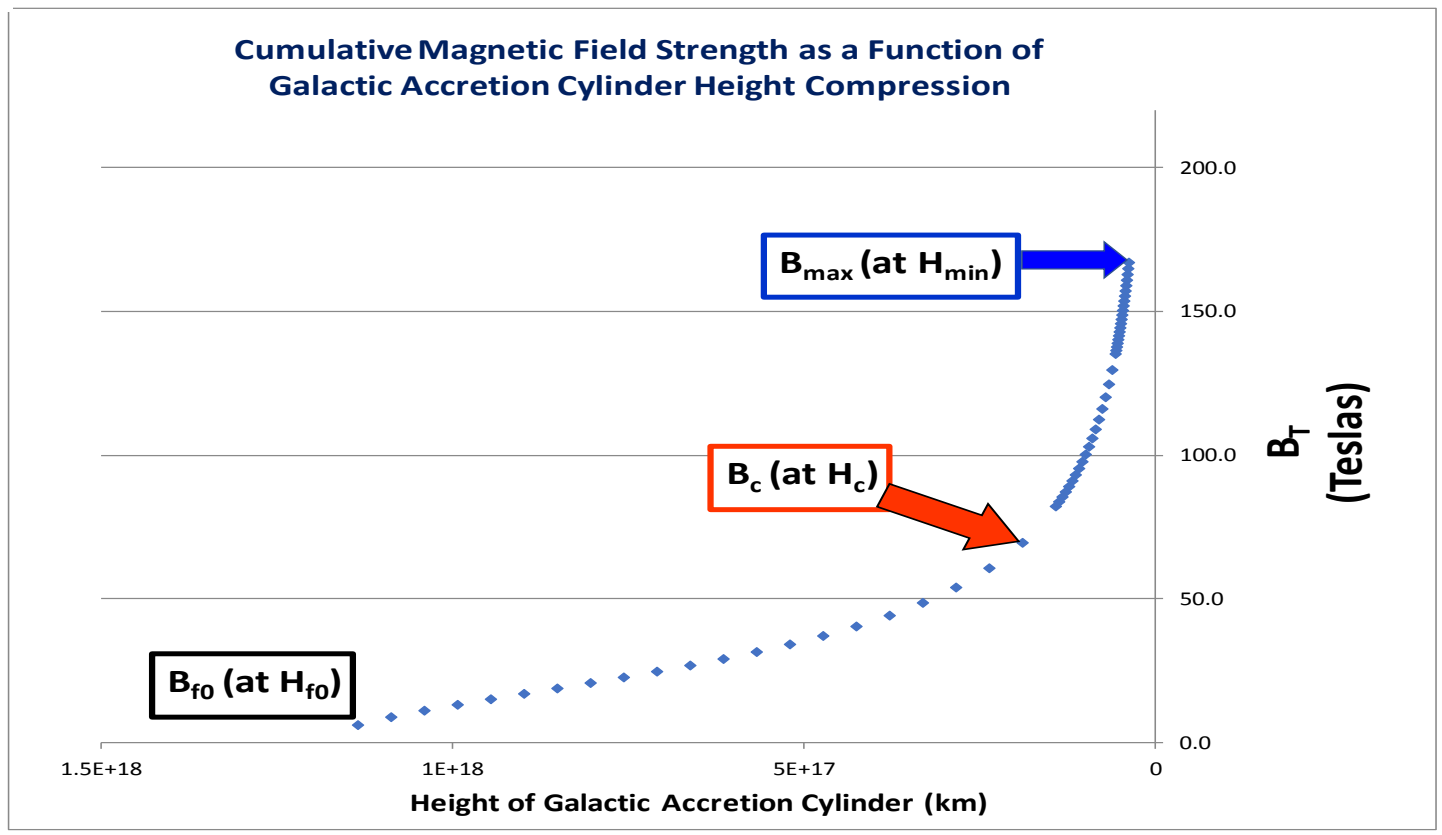

Figure 7B: Relative Magnetic Field $B_{T} / B_{\text {sun }}$ During the Formation Process of the Milky Way Galactic Core

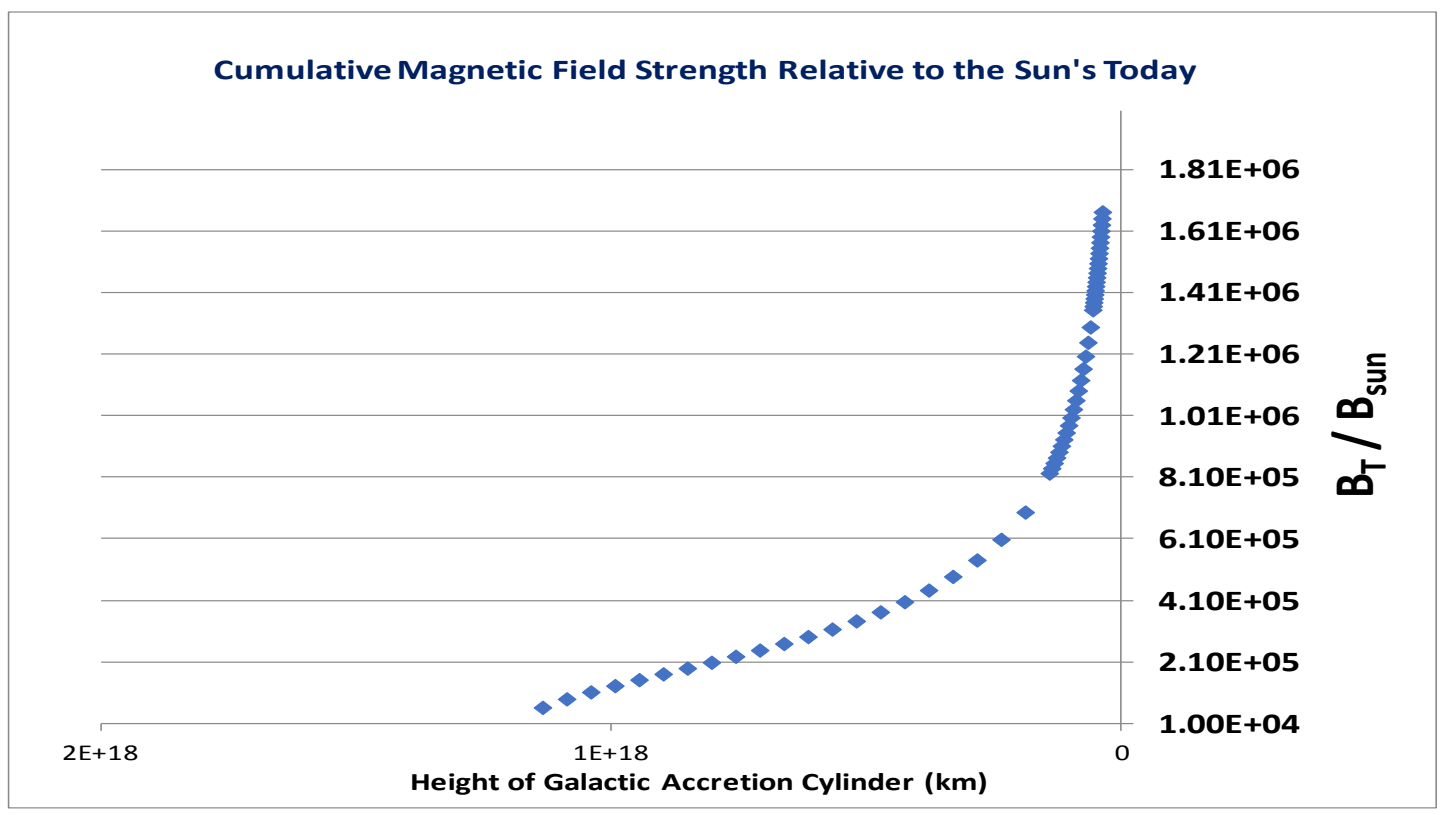

As seen in Figure 7B, the magnitude of the magnetic field during the formation process of the Milky Way's core region is predicted to have grown from 10,000 times (at the start of Stage 3) to $\sim 1.7$ million times greater (at the end of Stage 3 of formation) relative to the Sun's current 
magnetic field. Considering that the Stage 3 process of the proposed NOEMKV-field galactic model would likely have taken tens (or hundreds) of millions of years, this would likely fulfill the incredibly intense total magnetic field (magnetic pressure force) effect needed for shaping such huge amounts of mass with such colossal kinetic energy into the familiar $\sim 2 \mathrm{D}$ planar shape of the Milky Way and many similar galaxies.

The cumulative magnitude of the magnetic field of the Milky Way model relative to the starformation model of our Sun (from the previous publication) are very similar. This is not surprising because the state of matter (density as a function of height) and compressibility (bulk modulus as a function of density) are assumed to be the same. The key differences are:

- The models calculate volume-compression based on height-compression; assigning a time-frame over which the height-compression occurs has not yet been attempted. Considering the mind-boggling amount of mass and size, it is likely that the Milky-Way formation would have taken considerably longer than a star-formation. While the general shape of the cumulative magnetic fields vs height would be retained, the slope of $\mathrm{B}_{\mathrm{T}} \mathrm{vs} \mathrm{H}$ would likely be different when plotted on a time-axis in place of a height-axis.

- The cumulative magnetic pressure is of course force per unit area, as for any pressure. The area over which the magnetic pressure acts in galaxy formation compared to the starformation model leads to considerably more epic total magnetic force from the equations:

$\boldsymbol{F}_{\boldsymbol{B}}=\mathbf{2} *\left(\boldsymbol{\pi} * \boldsymbol{R}_{\boldsymbol{t}}{ }^{2}\right) * \mathbf{P}_{\mathbf{m c}}$ (Galactic Model)

$\boldsymbol{F}_{\boldsymbol{B}}=\mathbf{2} *\left(\boldsymbol{\pi} *\left(\boldsymbol{R}_{\boldsymbol{t}}{ }^{2}-\mathbf{R}_{\mathbf{i}}^{\mathbf{2}}\right)\right) * \mathbf{P}_{\mathbf{m c}}$ (Star-Formation Model)

Where:

$\mathbf{F}_{\mathbf{B}}=$ the cumulative magnetic pressure force (Newtons) acting on the top and bottom faces of the accretion cylinder matter, in the $+\mathrm{Z}$ and $-\mathrm{Z}$ axis

$\mathbf{R}_{\mathbf{t}}=$ total radius of the accretion cylinder

$\mathbf{R}_{\mathbf{i}}=$ inner radius of the accretion cylinder (eye of the fire-tornado vortex)

$\mathbf{P}_{\mathbf{m c}}=$ the cumulative magnetic pressure (in $\mathrm{N} / \mathrm{m}^{2}$ ) summed over each stage of compression

The factor of " 2 " appears in both equations because the pressure acts on both the top and bottom faces of the accretion cylinder. For the galactic model it is assumed that $R_{i}$ (radius of the black hole / eye of the vortex) is very small relative to $R_{t}$ of the galactic core vortex as in the case of the Milky Way.

Tabulating the results at the end of Stage 3 of formation (the peak of the NOEMKV field strength) and comparing them to the star-formation model for our Sun yields:

Table 2:

\begin{tabular}{|c|c|c|}
\hline Formation Model & $\begin{array}{c}\text { Total Area of Top and Bottom } \\
\left.\text { Faces of Accretion Cylinder } \mathbf{( m}^{\mathbf{2}}\right)\end{array}$ & $\mathbf{F}_{\mathbf{B}}$ (Newtons) \\
\hline Galactic- Milky Way & $1.265 \mathrm{E} 39$ & $1.409 \mathrm{E} 49$ \\
\hline Star- Model 1 of our Sun & $4.257 \mathrm{E} 19$ & $4.785 \mathrm{E} 29$ \\
\hline Star- Model 2 of our Sun & $4.257 \mathrm{E} 19$ & $5.965 \mathrm{E} 29$ \\
\hline
\end{tabular}


Taking the relative ratios implies that the magnetic force at the peak NOEMKV field strength during formation of the Milky Way would be about 26 billion billion times greater than the peak NOEMKV field strength during the formation of our Sun.

The final component of this phase of the modeling is the direction of the electric field and spawned magnetic field / magnetic pressure. The constraints utilized are:

- The bulge of the Milky Way's galactic core appears to be a flattened sphere, with the outer-most diameter of the sphere being compressed into the depth $(\sim 3,500 \mathrm{LY})$ of the thick-disk ${ }^{8}$

- The current galactic magnetic field measured at the surface of the Earth is only $10^{-6}$ that of the Earth's 9

Because the EM field involves convective motion of the cylindrical shells (Figures 1 \& 2) in 4-D space-time, this modeling is best demonstrated with computer animation. This is being developed in the Unity platform. The general concept is approximated in Figure 8 for ChargeDistribution Model 2:

Figure 8: Approximation of net-vector of magnetic field from the top and bottom boundary conditions of Charge-Distribution Model 2

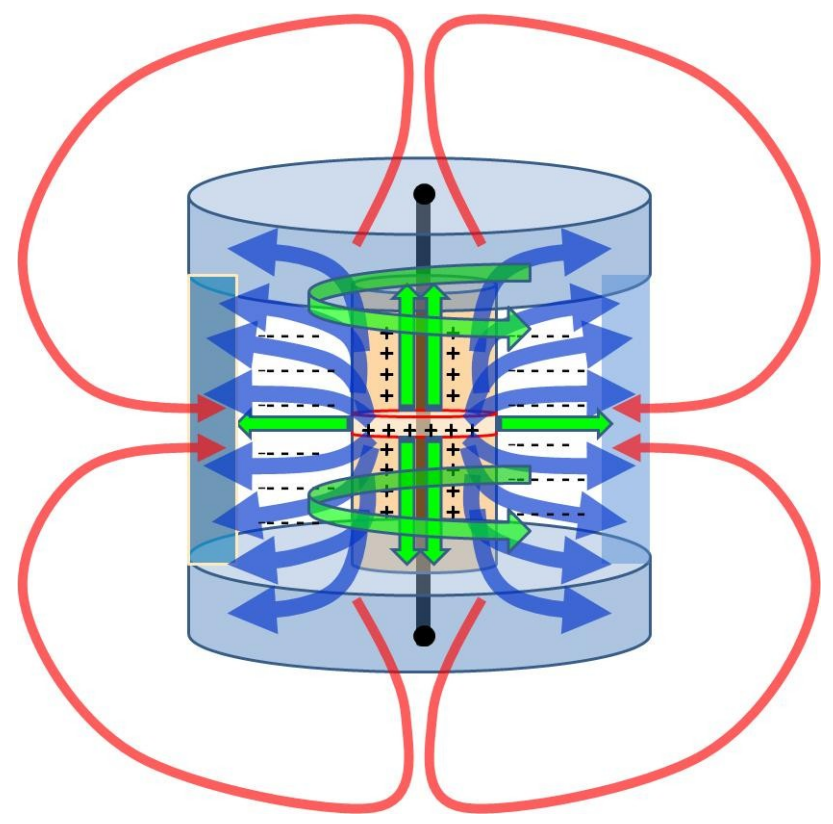

The magnetic field lines (red arrows) for Charge-Distribution Model 2 at the upper and lower boundary conditions would start as shown due to the transverse electric field along the eye of the vortex (the black hole) and then bend sharply back toward the sidewalls of the accretion cylinder due to curvature of the net-vector of electric field ((blue arrows) and the strongly-negative cylindrical shell around the strongly-positive inner cylindrical shell. The mid-point region of the $\mathrm{Z}$ axis is proposed to serve as the "South Pole" where magnetic field lines above this region form lobes that converge in the $-\hat{Z}$ direction and magnetic field lines below this region converge in the $+\hat{Z}$ direction. The net magnetic pressure vector of the net-magnetic field line vector would mostly be in the $-Z$ direction (from the magnetic field lines above the "Z-central Pole") and in the $+Z$ direction (from the magnetic field lines below the "Z-central Pole"). 
Figure 8 is for any instantaneous moment in time though will hold true in dynamic 4-D space time with the rotating vortex of the accretion cylinder (and the rotating charge-distribution cylindrical shells) that is predicted to organize and strengthen the electromagnetic fields further with convection-displacement currents azimuthally about the $\hat{Z}$-axis; this will be demonstrated in the computer animations. The mentioned constraints are satisfied with the vectors of Figure 8 via:

- The flattened spherical shape of the Milky Way's galactic core "bulge" are explained by the curvature of the magnetic field lines (and associated orthogonal magnetic pressure vector) compressing the outer edges of the galactic core to a cylinder with uniformly flattened outer edges (that will appear as a flattened sphere when viewed from the side)

- The observed measurements of negligible galactic magnetic field detected on Earth are satisfied by the same curvature confining the magnetic field largely to the galactic-core region

- The magnetic field detected on Earth would probably have been considerably stronger billions of years ago during the galactic-core formation process; it is postulated that much more of our galaxies matter was likely part of the hot primary galactic vortex- rotating as free discrete matter (and thereby increasing the size and strength of the NOEMKV field forces and distance over which they acted) before being ejected / spun out of the galactic core to coalesce into billions of stars and celestial objects of the Milky Way

The net-effect of the NOEMKV field vectors during the formation process of the Milky Way and similar galaxies is the compressing of rotating matter into the familiar $\sim 2 \mathrm{D}$ planar celestial kinematics we observe today. As with the solar-formation models, the main work of the proposed colossal electromagnetic field / magnetic pressure in shaping the kinematics of orbital shapes and geometries into $\sim 2 \mathrm{D}$ planar would have already been completed during the galactic formation process and conservation of angular momentum (imparted by the fire-tornado vortex) thereafter. The postulate that a majority of the Milky Way's matter was concentrated in and around the primary galactic core vortex during the formation period such that the epic magnetic pressure of the NOEMKV field imparted the $\sim 2 \mathrm{D}$ planar kinematics to all of the matter that eventually became billions of stars orbiting the core in this plane will be modeled in more detail in subsequent study.

\section{Authors' notes:}

This paper is a continuation of the father-son team research and computational modeling started with our star-formation modeling. The content is the foundation of co-author Henry Bushong's senior year scientific investigation project; his specialty is computer programming and he has written algorithms in $\mathrm{C} \#$ for the computations thus far and continues to work on the 4-D spacetime coded simulations as a visual demonstration tool for the concepts.

We recently came upon an article on nuclear fusion and the difficulties that arise in handling plasma. To control unpredictable and chaotic forces of nature requires one or more forces that are sufficiently powerful and directionally-focused enough to make it predictable; if our astrophysics theories are correct in that the magnetic pressure generated by rotating vortex celestial accretion matter of gas/plasma/dust is powerful enough to organize and compress that accretion matter (much of which was plasma during the formation process) to the familiar $\sim 2 \mathrm{D}$ 
planar celestial kinematics of enormous objects (stars, planets, and galaxies) over enormous distances and contrary to the law of entropy favoring random 3D (4D with time) spatial distribution, these concepts should be adaptable to controlling plasma for humanity's use at appropriate scale. We hope that the vortex-organization and subsequent well-organized and strong electric field, magnetic field, and direction of magnetic pressure of the proposed NOEMKV field models could be part of the solution to the plasma problems and help humanity achieve nuclear fusion as a clean-energy source.

Anyone wishing to add insights or parameter data is welcome to do so by contacting: M2Materials@,Outlook.com

All original content herein (C) James and Henry Bushong

\section{References}

${ }^{1}$ Bushong, H., Bushong, J. Theories on the Formation and Evolution of 2 D Planar Celestial Kinematics OSF Preprints

${ }^{2}$ https://solarsystem.nasa.gov/resources/285/the-milky-way-galaxy/

3 https://sites.astro.caltech.edu/ george/ay20/Chiappini-MilkyWay.pdf Chiappini, C., American Scientist, November/December 2001, pp. 506-515. (C) 2001 by American Scientist

${ }^{4}$ https://astronomy.com/news/2020/04/how-much-space-does-a-black-hole-take-up

5 Thakor P.B. et al, Bulk Modulus of Some Simple Liquid Metals, Indian Journal of Pure \& Applied Physics (Vol. 46, June 2008, p. 431-434)

${ }^{6}$ Waseda Y \& Jacob KT, Phys Status Solidi, 68 (1981), K117

${ }^{7} \mathrm{https}: / /$ farside.ph.utexas.edu/teaching/plasma/lectures/node63.html

8 https://sites.astro.caltech.edu/ george/ay20/Chiappini-MilkyWay.pdf Chiappini, C., The Formation and Evolution of the Milky Way, American Scientist, November/December 2001, pp. 506-515. (C) 2001 by American Scientist ${ }^{9}$ https://www.britannica.com/place/Milky-Way-Galaxy/The-structure-and-dynamics-of-theMilky-Way-Galaxy 